\title{
THERMOACOUSTIC MODES OF QUASI-1D COMBUSTORS IN THE REGION OF MARGINAL STABILITY
}

\author{
Camilo F. Silva*, Kah Joon Yong \\ Professur für Thermofluiddynamik \\ Technische Universität München \\ Email: silva@tfd.mw.tum.de
}

\author{
Luca Magri \\ University of Cambridge \\ Engineering Department \\ Cambridge, UK
}

\begin{abstract}
It may be generally believed that the thermoacoustic eigenfrequencies of a combustor with fully acoustically reflecting boundary conditions depend on both flame dynamics and geometry of the system. In this work, we show that there are situations where this understanding does not strictly apply.

The purpose of this study is twofold. In the first part, we show that the resonance frequencies of two premixed combustors with fully acoustically reflecting boundary conditions in the region of marginal stability depend only on the parameters of the flame dynamics, but do not depend on the combustor's geometry. This is shown by means of a parametric study, where the time delay and the interaction index of the flame response are varied and the resulting complex eigenfrequency locus is shown. Assuming longitudinal acoustics and a low Mach number, a quasi-1D Helmholtz solver is utilized. The time delay and interaction index of the flame response are parametrically varied to calculate the complex eigenfrequency locus. It is found that all the eigenfrequency trajectories cross the real axis at a resonance frequency that depends only on the time delay. Such marginally stable frequencies are independent of the resonant cavity modes of the two combustors, i.e. the passive thermoacoustic modes.

In the second part, we exploit the aforementioned observation to evaluate the critical flame gain required for the systems to become unstable at four eigenfrequencies located in the marginally stable region. A computationally-efficient method is proposed. The key ingredient is to consider both direct and
\end{abstract}

*Address all correspondence to this author. adjoint eigenvectors associated with the four eigenfrequencies. Hence, the sensitivity of the eigenfrequencies to changes in the gain at the region of marginal stability is evaluated with cheap and accurate calculations.

This work contributes to the understanding of thermoacoustic stability of combustors. In the same manner, the understanding of the nature of distinct resonance frequencies in unstable combustors may be enhanced by employing the analysis of the eigenfrequency locus here reported.

\section{INTRODUCTION}

Thermoacoustic instabilities in combustion chambers of gas turbines and rockets are persistent and intractable problems that industry faces. Such instabilities are due to the two-way coupling between the unsteady heat release rate of the flame and the acoustic waves originated by the volumetric expansion in the reactive region $[1,2]$. If a thermoacoustic instability is triggered, large pressure oscillations arise, which may lead to critical failure of the system. One well-known technique for the study of thermoacoustic instabilities is based on a divide-and-conquer approach. First, the flame response to incoming acoustic perturbations is evaluated either experimentally or numerically [3-6]. Second, the acoustics is calculated by Helmholtz solvers, or linearized Navier-Stokes equations, or lower-order methods, such as acoustic network models [7-10]. The flame response is thus introduced in the acoustic equations as a source term. This results in an eigenvalue problem, where the eigenfrequencies typ- 
ically appear under nonlinear terms. The eigenfrequency provides the resonance frequency and growth rate of the thermoacoustic modes of interest. Attention is generally given to unstable modes, which have positive growth rate, or marginally stable modes, which have zero growth rate. Importantly, choosing an accurate model for the system's damping is essential for a correct estimation of the linear stability and limit-cycle oscillations at the marginally stable region $[9,11]$

Thermoacoustic systems with fully or partially acoustically reflecting boundary conditions that do no contain any flame dynamics (the gain of the flame response being zero) are characterized by passive thermoacoustic modes. (The mean temperature and density are, however, still affected by the flame.) For example, the acoustics of a duct with Neumann (closed inlet) and Dirichlet (open outlet) boundary conditions at low frequency is characterized by a quarter-wave thermoacoustic mode. When the dynamics of the flame is taken into account, this mode may be enhanced or suppressed by the interaction with the heat release rate generated by flame. When the flame response is modeled by an $n-\tau$ model $[12,13]$, the thermoacoustic system is stable when the time delay $\tau$ is smaller than half of the thermoacoustic period [14]. Thus, considering both the flame dynamics, here described by the $n-\tau$ model, and the system's acoustics, here characterized by the period of the quarter-wave thermoacoustic mode, is essential for the assessment of the thermoacoustic stability of the system.

Over the last few years, the understanding of thermoacoustic stability has been improved by the discovery of Intrinsic ThermoAcoustic (ITA) instabilities [6,15-17]. It was found that systems with anechoic boundary conditions, which do not have passive thermoacoustic modes depending on the system's geometry, can be thermoacoustically unstable. The physical causes that bring about ITA feedback loop is different from other thermoacoustic instabilities: Velocity fluctuations upstream of the flame perturb the flame dynamics, hence, the heat release rate fluctuate generating further acoustic waves. The acoustic waves that travel upstream interfere with the velocity fluctuations at the flame's base, which closes the ITA feedback loop. If the interference is constructive, ITA instabilities arise. Based on a $n-\tau$ flame response model, a stability criterion was obtained for anechoic systems by Hoeijmakers et al. [15] and Emmert et al. [16]. It was found that ITA modes, which are characterized by a resonance frequency $f=j /(2 \tau)$ (where $j=1,3,5, \cdots$ is an odd number) are unstable if the interaction index is larger than a critical value, which is a function of the temperature ratio. By investigating a turbulent swirled combustor with partially reflecting boundary conditions and a frequency-dependent flame response, Silva et al. [18] subsequently found that the ITA feedback loop plays an important role not only in the stability of the system but also in the generation of combustion noise. Moreover, when considering systems with fully reflecting conditions, it was found by Mukherjee and Shrira [19] that the ITA resonance frequencies at the neutral curve (when the growth rate is exactly zero) is the same as the one associated with the ITA modes in anechoic systems $f=j /(2 \tau)$. Therefore, it was concluded that the resonance frequency at the neutral curve of the ITA modes in systems with fully reflecting acoustic boundary conditions is independent of the system's geometry.

Therefore, ITA instabilities may be present in systems with anechoic, partial or fully reflecting boundary conditions.

In this paper, it is shown that, except for passive thermoacoustic modes, all resonance frequencies at the neutral curve, within the frequency band considered, depend exclusively on the flame dynamics and do not depend on the geometry. This is shown by considering thermoacoustic systems with fully acoustically reflecting boundary conditions. These modes, which are ITA modes because of their exclusive dependence on the flame dynamics, are the only (active) thermoacoustic modes at the neutral curve. Importantly, the stability of ITA modes is strongly related to a critical gain. A computationally-efficient methodology is proposed to accurately calculate the critical gain of an ITA thermoacoustic mode. By analyzing the structure of both direct and adjoint thermoacoustic modes at the marginally stable region, we explain why some configurations exhibit critical gains that are much larger (or smaller) than other configurations. The methodology proposed is applied to combustors with threecoaxial ducts and a compact flame. It can be applied to any type of combustor's geometry when a stability solver is available to build the eigenvalue problem.

The paper is organized as follows. In the next section the quasi-1D Helmholtz equation is derived. Subsequently, two premixed combustors are investigated. A locus of the eigenfrequencies, which results from performing systematic variations of two parameters of the flame response, is computed by solving the nonlinear eigenvalue problem corresponding to the Helmholtz equation. Afterwards, a method is proposed to compute in an efficient way the direct and adjoint eigenvectors, in addition to the critical gain and corresponding sensitivity at the neutral curve. Finally, the obtained results are presented and the thermoacoustic stability of the two combustors investigated is analyzed.

\section{THE QUASI-1D HELMHOLTZ EQUATION}

Since we are interested in thermoacoustic systems with frequencies that are smaller than the cut-on frequency of transversal modes, we focus on quasi-1D systems, where only longitudinal acoustic modes are modeled. Additionally, if a low Mach number flow is considered, the momentum and energy conservation 
equations read, respectively

$$
\begin{aligned}
\bar{\rho} \frac{\partial u^{\prime}}{\partial t}+\frac{\partial p^{\prime}}{\partial x} & =0 \\
\frac{S}{\bar{\rho} \bar{c}^{2}} \frac{\partial p^{\prime}}{\partial t}+\frac{\partial}{\partial x}\left(S u^{\prime}\right) & =\frac{(\gamma-1)}{\bar{\rho} \bar{c}^{2}} \dot{q}^{\prime} S
\end{aligned}
$$

where $u, p$ and $\dot{q}$ denote velocity, pressure and heat release rate. The quantities $\rho, c, \gamma$ and $S$ stand for density, speed of sound, heat capacity ratio and cross-sectional area, respectively. The symbols [] and [ ]' represent mean and fluctuating quantities. Subtracting the spatial derivative of Eqn. (1) from the temporal derivative of Eqn. (2) results in

$$
\frac{1}{S} \frac{\partial}{\partial x}\left(S \bar{c}^{2} \frac{\partial \hat{p}}{\partial x}\right)+\omega^{2} \hat{p}=-i \omega(\gamma-1) \hat{\dot{q}}
$$

where the harmonic transformation []$^{\prime}=\left[\hat{]} e^{i \omega t}\right.$ has been applied. By assuming that the flame is acoustically compact, i.e. the flame length is negligible with respect to the acoustic wavelength, the local heat release rate $\hat{\dot{q}}$ can be expressed in terms of the global heat release rate $\hat{\dot{Q}}$ as $\hat{\dot{q}}=I_{\mathrm{d}} \hat{\dot{Q}} / V_{\mathrm{f}}$, where $V_{\mathrm{f}}$ is the volume of the flame [9]. $I_{\mathrm{d}}$ is one in the flame area and zero elsewhere. Upon numerical discretization, $I_{\mathrm{d}}$ represents a Kronecker delta. We express now $\hat{\dot{Q}}=\hat{\dot{Q}}_{\mathrm{c}}+\hat{\dot{Q}}_{\mathrm{n}}$, where $\hat{\dot{Q}}_{\mathrm{c}}$ represents the heat release rate coupled with upstream velocity perturbations and $\hat{\dot{Q}}_{\mathrm{n}}$ denotes the heat release rate correlated to turbulent fluctuations. By considering that $V_{\mathrm{f}}=S_{\mathrm{f}} l_{\mathrm{f}}$, where $l_{\mathrm{f}}$ is a characteristic flame length, Eqn. (3) becomes

$$
\begin{array}{r}
\frac{1}{S} \frac{\partial}{\partial x}\left(S \bar{c}^{2} \frac{\partial \hat{p}}{\partial x}\right)+\omega^{2} \hat{p}+i \omega(\gamma-1) I_{\mathrm{d}} \hat{\dot{Q}}_{\mathrm{c}} /\left(S_{\mathrm{f}} l_{\mathrm{f}}\right) \\
=-i \omega(\gamma-1) I_{\mathrm{d}} \hat{\dot{Q}}_{\mathrm{n}} /\left(S_{\mathrm{f}} l_{\mathrm{f}}\right) .
\end{array}
$$

The heat release rate associated to $\hat{\dot{Q}}_{\mathrm{c}}$ can be related to upstream velocity perturbations at a reference position $\hat{u}_{\text {ref }}$ by means of the flame transfer function $\mathscr{F}(\omega)$, defined as

$$
\frac{\hat{\dot{Q}}_{\mathrm{c}}}{\overline{\dot{Q}}}=\mathscr{F}(\omega) \frac{\hat{u}_{\mathrm{ref}}}{\bar{u}_{\mathrm{ref}}}
$$

We express now the mean heat release rate as $\overline{\dot{Q}}=$ $\bar{\rho}_{\text {ref }} \bar{u}_{\text {ref }} S_{\text {ref }} c_{\mathrm{p}} T_{\mathrm{u}} \theta$, where $c_{\mathrm{p}}$ is the specific heat capacity at constant pressure and $\theta=T_{\mathrm{d}} / T_{\mathrm{u}}-1$ is the relative temperature increment across the flame. The indices ' $u$ ' and ' $d$ ' stand for 'upstream' and 'downstream' (of the flame), respectively. Thus, the quantity $i \omega(\gamma-1) \hat{\dot{Q}}_{\mathrm{c}}$ can be expressed as

$i \omega(\gamma-1) \hat{\dot{Q}}_{\mathrm{c}}=i \omega \bar{\rho}_{\mathrm{ref}} \bar{c}_{\mathrm{ref}}^{2} S_{\mathrm{ref}} \theta \mathscr{F}(\omega) \hat{u}_{\mathrm{ref}}=-\left.\bar{c}_{\mathrm{ref}}^{2} S_{\mathrm{ref}} \theta \mathscr{F}(\omega) \frac{\partial \hat{p}}{\partial x}\right|_{\mathrm{ref}}$,

where the momentum equation (1), re-written as $\hat{u}=\frac{-1}{i \omega \bar{\rho}} \frac{\partial \hat{p}}{\partial x}$, has been considered. Finally, the Helmholtz equation for quasi-1D acoustic systems reads

$\underbrace{\frac{1}{S} \frac{\partial}{\partial x}\left(S \bar{c}^{2} \frac{\partial \hat{p}}{\partial x}\right)}_{\mathscr{A} \hat{p}}-\underbrace{\left.K \mathscr{F}(\omega) \frac{\partial \hat{p}}{\partial x}\right|_{\mathrm{ref}}}_{\mathscr{H} \mathscr{F}(\omega) \hat{p}}+\underbrace{\omega^{2} \hat{p}}_{-\sigma \hat{p}}=-i \omega(\gamma-1) \hat{\dot{Q}}_{\mathrm{n}} /\left(S_{\mathrm{f}} l_{\mathrm{f}}\right)$

where $K=I_{\mathrm{d}} \alpha \theta \bar{c}_{\text {ref }}^{2} / l_{\mathrm{f}}$ and $\alpha=S_{\text {ref }} / S_{\mathrm{f}}$. In the present study, we are interested in the Helmholtz equation and the corresponding eigenvalue problem. After dropping the forcing term from the formulation, the eigenvalue problem obtained reads

$$
\left(\mathscr{A}-\mathscr{H} \mathscr{F}\left(\omega_{k}\right)\right) \hat{p}_{k}=\sigma_{k} \hat{p}_{k} \quad \Rightarrow \quad \mathscr{L} \hat{p}_{k}=\sigma_{k} \hat{p}_{k}
$$

where $\hat{p}_{k}$ denotes the $k_{\mathrm{th}}$ acoustic mode and $\omega_{k}$ is the corresponding complex eigenfrequency, which in turn is linked to the $k_{\mathrm{th}}$ eigenvalue by $\sigma_{k}=-\omega_{k}^{2}$. In the present work, the nonlinear eigenvalue problem of Eqn. (8) is solved by means of an in-house Helmholtz solver based on a finite-difference numerical scheme. A methodology based on a fixed-point iteration with relaxation, which is similar to that of Silva et al. [9], was implemented.

\section{THE LOCUS OF THE EIGENFREQUENCIES AROUND THE FIRST LONGITUDINAL ACOUSTIC MODE}

One of the objectives of this work is to understand the structure of both ITA and classical thermoacoustic modes at the marginal region of stability. Before performing such an analysis, we plot the locus of the eigenfrequencies given by Eqn. (8) around the first passive thermacoustic mode. The analysis can be performed around any other passive thermoacoustic mode.

\section{Two premixed combustors}

Two different configurations are studied. The first configuration (Duct), is a duct flame previously studied in the work of Hoeijmakers et al. [15]. The second configuration (BRS) is a premixed swirled combustor previously studied in the works of $[5,18,20,21]$. Figure 1 illustrates these two configurations, and Tab. 1 shows the geometric and thermodynamic parameters of interest. In this study, the inlet boundary condition is of Neumann type, $\partial \hat{p} / \partial x=0$, whereas the outlet is of Dirichlet type, $\hat{p}=0$.

The first passive thermoacoustic mode $\hat{p}_{1 p}$ (Fig. 2) and 


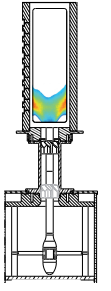

BRS

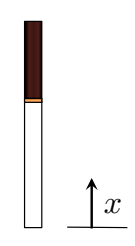

Duct

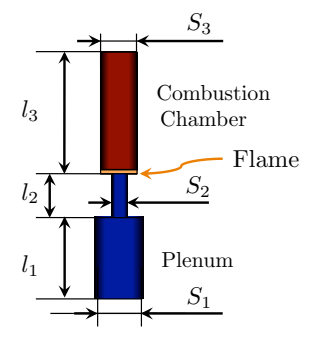

FIGURE 1. The two configurations under investigation. Corresponding dimensions and thermodynamics parameters are given in Tab. 1.

TABLE 1. Geometric and thermodynamic parameters of the two configurations under investigation.

\begin{tabular}{|l|l|l|}
\hline Parameter & BRS & Duct \\
\hline$l_{1}(\mathrm{~m})$ & 0.17 & 0.15 \\
$l_{2}(\mathrm{~m})$ & 0.18 & 0.15 \\
$l_{3}(\mathrm{~m})$ & 0.336 & 0.2 \\
$S_{1}\left(\mathrm{~m}^{2}\right)$ & $\pi / 40.2^{2}$ & 1 \\
$S_{2}\left(\mathrm{~m}^{2}\right)$ & $\pi / 4\left(0.04^{2}-0.016^{2}\right)$ & 1 \\
$S_{3}\left(\mathrm{~m}^{2}\right)$ & $0.09^{2}$ & 1 \\
\hline $\bar{T}_{\mathrm{u}}(\mathrm{K})$ & 293 & 293 \\
$\bar{T}_{\mathrm{d}}(\mathrm{K})$ & 1930 & 1600 \\
$\bar{\rho}_{\mathrm{u}}\left(\mathrm{kg} / \mathrm{m}^{3}\right)$ & 1.204 & 1.204 \\
$\bar{\rho}_{\mathrm{d}}\left(\mathrm{kg} / \mathrm{m}^{3}\right)$ & 0.183 & 0.22 \\
$\bar{c}_{\mathrm{u}}(\mathrm{m} / \mathrm{s})$ & 343 & 343 \\
$\bar{c}_{\mathrm{d}}(\mathrm{m} / \mathrm{s})$ & 881 & 774 \\
\hline
\end{tabular}

the corresponding eigenfrequency $\omega_{1 p}$ are obtained by solving Eqn. (8) with $\mathscr{F}(\omega)=0$. On one hand, the first thermoacoustic mode of the 'Duct' configuration is associated with the quarterwave mode of the duct with $\omega_{1 p}=2 \pi \cdot 250 \mathrm{rad} / \mathrm{s}$. On the other hand, the first thermoacoustic mode of the 'BRS' configuration is the Helmholtz mode of the plenum with $\omega_{1 p}=2 \pi \cdot 55 \mathrm{rad} / \mathrm{s}$.

\section{The loci of eigenfrequencies}

In this study, the flame response is modeled by an $n-\tau$ model so that $\mathscr{F}(\omega)=n e^{-i \omega \tau}$, where $n$ is the interaction index and $\tau$ denotes a characteristic time delay. Note, that a more realistic flame response should consider the dependence of $n$ on the frequency. However, the $n-\tau$ model is used in this study to capture the essential features of the flame response. We carry out a

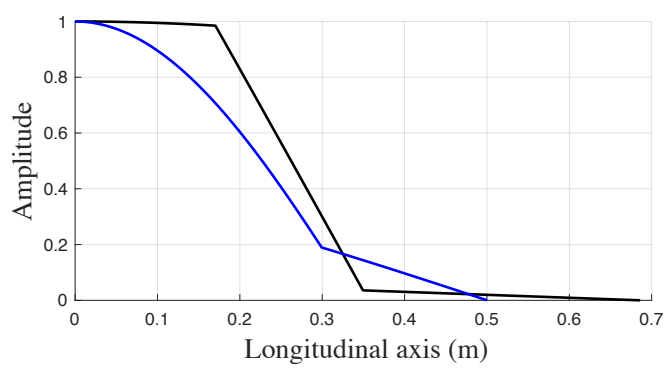

FIGURE 2. First passive thermoacoustic mode $\hat{p}_{1 p}$. (Blue) Duct, (Black) BRS.

parametric study by varying $n=[0 \rightarrow 1]$ for the Duct configuration and $n=[0 \rightarrow 4]$ for the BRS configuration. The time delay is varied as $\tau=\left[0 \rightarrow 2 \pi / \omega_{1 p}\right]$ for both configurations. The corresponding eigenfrequencies, which are solutions of Eqn. (8), are plotted in the complex plane. Figures 3 and 4 show the results for the Duct configuration and 'BRS' combustor, respectively.

Although the two configurations are different, the loci obtained have strong similarities. In both Duct and BRS cases we observe a star-like structure and curvilinear trajectories. The center of the star is given by the eigenfrequency of the passive acoustic mode $\omega_{1 p}$. Once $n$ is increased from zero, the eigenfrequencies depart from $\omega_{1 p}$. These trajectories rotate counter clock-wise as $\tau$ increases. We can observe that some trajectories becomes unstable (growth rate $>0$ ) for $n>n_{g_{0}}$, where $n_{g_{0}}$ denotes the critical interaction index.

Let us now concentrate on the trajectories connected to the star center $\omega_{1 p}$ and defined by $\tau=m \cdot 2 \pi / \omega_{1 p}$ with $0.32<m<$ 0.33 (dashed lines in Figs. 3 and 4). We observe that these trajectories change direction suddenly (from left to right when increasing $m$ ) after a critical value $m_{\mathrm{c}} \approx 0.325$, which corresponds to $\tau_{\mathrm{c}} \approx 1.3 \mathrm{~ms}$ for the Duct configuration and $\tau_{\mathrm{c}} \approx 5.9 \mathrm{~ms}$ for the BRS combustors. The intersection of the trajectory $m_{\mathrm{c}} \approx 0.325$ is a defective eigenfrequency, which has double algebraic multiplicity but only one associated thermoacoustic mode (result not shown). Although stable, this point has high sensitivity to small changes of $n$ and $\tau$.

From Figs. 3 and 4 it is possible to observe quasi-vertical trajectories, the growth rates of which tend to $-\infty$ as $n \rightarrow 0$. As observed also by Mukherjee and Shrira [19], the resonance frequency tends to $\operatorname{real}(\omega)=2 \pi \cdot j /(2 \tau)$ (with $j=1,3,5, \cdots)$ and $\operatorname{Imag}(\omega)=-\infty$ when $n$ approaches zero, as illustrated in Fig. (5) for the duct configuration. The real part of this frequency corresponds to ITA resonance frequencies $[15,16]$. Correspondingly, we label these trajectories as ITA trajectories.

The focus is now shifted to the resonance frequencies with zero growth rates (marginal stability). As already pointed out by Mukherjee and Shrira [19], this value is equal to $\operatorname{real}(\omega)=$ $2 \pi \cdot j /(2 \tau)$ for the ITA trajectories. In addition, from Figs. 3 and 


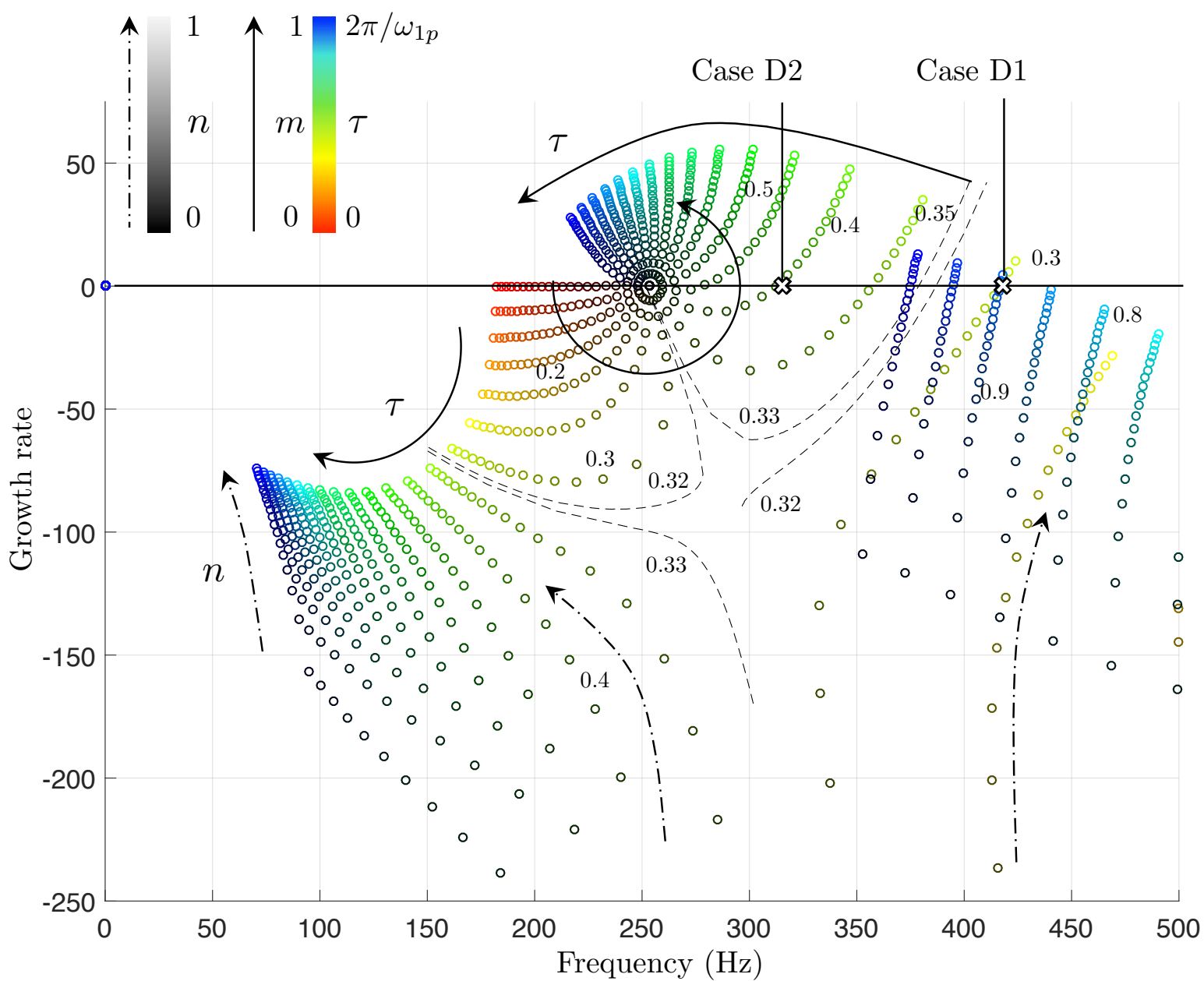

FIGURE 3. Locus of eigenfrequencies on the complex plane that corresponds to the Duct configuration. $n=[0 \rightarrow 1]$ with $\Delta n=0.05$ and $\tau=[0 \rightarrow$ $\left.2 \pi / \omega_{1 p}\right] . \tau=m \cdot 2 \pi / \omega_{1 p}$ with $m=0 \rightarrow 1$ and $\Delta m=0.05$. Numbers in the plot are values of $m$ for the closest trajectory. The growth rate is defined as $-\operatorname{Im}(\omega) / 2 \pi$.

4 it is observed that this is also the case for the trajectories that belong to the star-like structure and cross the real axis (for $m=$ 0.35, 0.4, 0.45). Indeed, it is observed in an extended plot (Fig. 5) that the resonance frequency at zero growth rate corresponding to all trajectories that cross the real-axis over the investigated range of $0<\operatorname{real}(\omega)<2 \pi \cdot 1000$ is a function exclusively of the time delay $\tau$. This finding is of interest for stability analysis of thermoacoustic systems since it establishes potential resonance frequencies in unstable combustors. As shown in the next section, this finding also allows us to efficiently compute the eigentriplet $\hat{p}_{k}, \hat{p}_{k}^{\dagger}, \omega_{k}$ at the neutral curve.

Solving the nonlinear eigenvalue problem (Eqn. (8)) to find ITA trajectories is numerically more challenging. It was neces- sary to apply small relaxation factors (around 0.005 ) to the fixedpoint algorithm to reach convergence.

\section{THE INTERACTION INDEX AT THE MARGINAL REGION OF STABILITY}

So far we have observed in Figs. 3 and 4 that all trajectories crossing the real axis exhibit a resonance frequency $\operatorname{real}(\omega)=$ $2 \pi \cdot j /(2 \tau)$ (with $j=1,3,5, \cdots)$ at zero growth rate. The purpose of this section is to show a computationally efficient technique that exploits this observation in order to compute the value of the interaction index $n_{g_{0}}$, which corresponds to the eigenfrequency $\omega_{g_{0}}=2 \pi \cdot j /(2 \tau)+i \cdot 0$. Subsequently, the sensitivity 


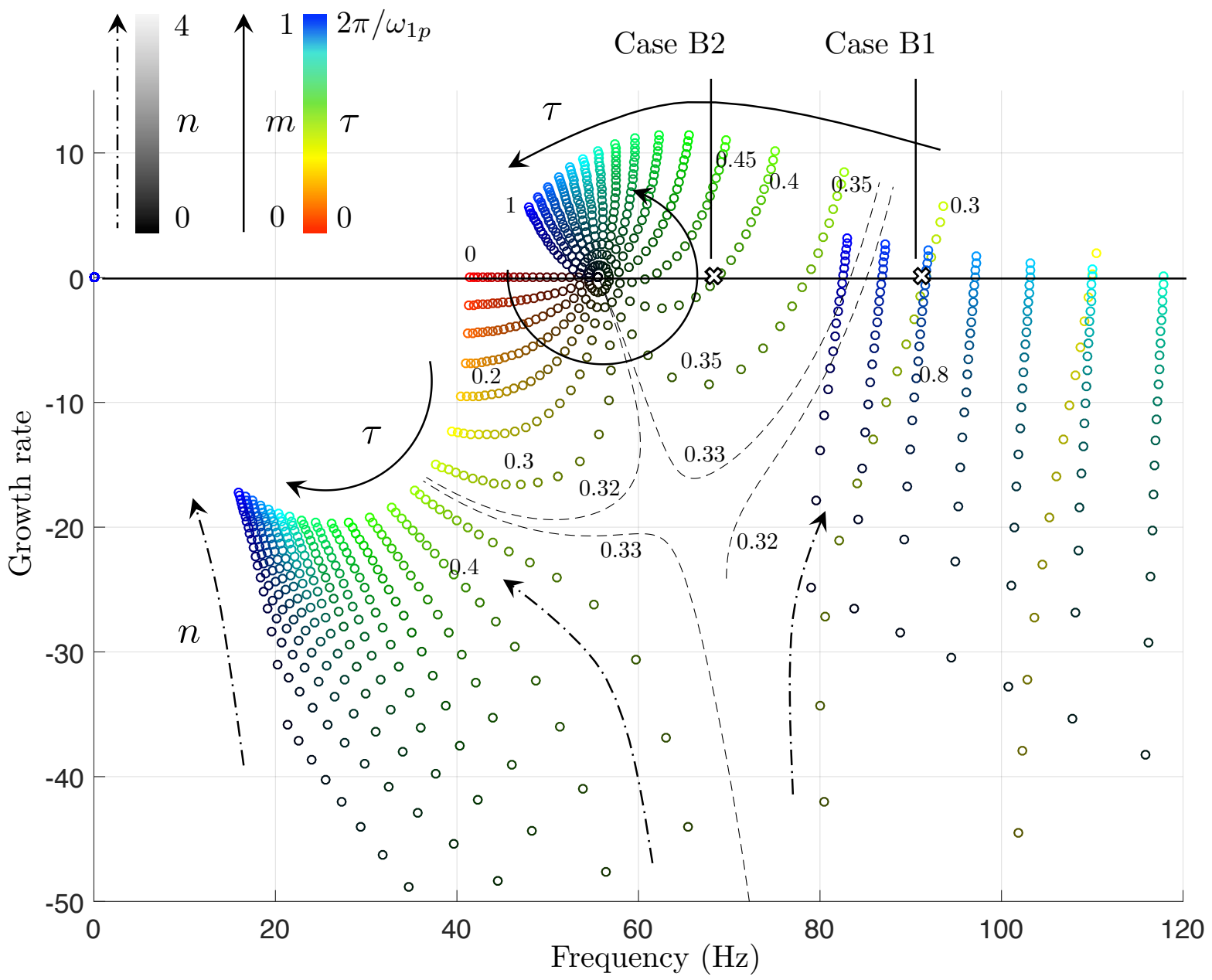

FIGURE 4. Locus of eigenfrequencies in the complex plane of the BRS configuration. $n=[0 \rightarrow 4]$ with $\Delta n=0.2$ and $\tau=\left[0 \rightarrow 2 \pi / \omega_{1 p}\right] . \tau=$ $m \cdot 2 \pi / \omega_{1 p}$ with $m=0 \rightarrow 1$ and $\Delta m=0.05$. Numbers in the plot are values of $m$ for the closest trajectory. The growth rate is defined as $-\operatorname{Im}(\omega) / 2 \pi$.

$\partial \omega /\left.\partial n\right|_{\omega=\omega_{g_{0}}}$ is evaluated with a computationally-efficient and accurate adjoint approach [22,23].

\section{The direct and adjoint eigenvectors}

The adjoint eigenvalue problem reads

$$
\mathscr{L}^{\dagger} \hat{p}_{k}^{\dagger}=\sigma_{k}^{\dagger} \hat{p}_{k}^{\dagger}
$$

where the symbol []$^{\dagger}$ denotes 'adjoint'. The adjoint eigenvalue $\sigma^{\dagger}$ is equal to the complex conjugate of $\sigma$, i.e. $\sigma^{\dagger}=\sigma^{*}$ and the adjoint operator $\mathscr{L}^{\dagger}$ is the conjugate transpose of $\mathscr{L}$, i.e. $\mathscr{L}^{\dagger}=\mathscr{L}^{H}$ [24]. Furthermore, for the biorthogonality condition, $\left\langle\hat{p}_{k}^{\dagger}, \hat{p}_{l}\right\rangle=\left(\hat{p}_{k}^{\dagger}\right)^{H} \hat{p}_{l}$, is equal to zero for $k \neq l$. The direct and adjoint eigenvectors are normalized such that $\left(\hat{p}_{k}^{\dagger}\right)^{H} \hat{p}_{k}=1$. Let us now project the direct eigenvalue problem (Eqn. (8)) for a given thermoacoustic mode $\hat{p}_{k}$ on the adjoint eigenvector $p_{k}^{\dagger}$. The new problem reads

$$
\left(\hat{p}_{k}^{\dagger}\right)^{H} \mathscr{L} \hat{p}_{k}=\left(\hat{p}_{k}^{\dagger}\right)^{H}\left(\mathscr{A}-\mathscr{H} n e^{-i \omega_{k} \tau}\right) \hat{p}_{k}=\sigma_{k}\left(\hat{p}_{k}^{\dagger}\right)^{H} \hat{p}_{k}=\sigma_{k} .
$$




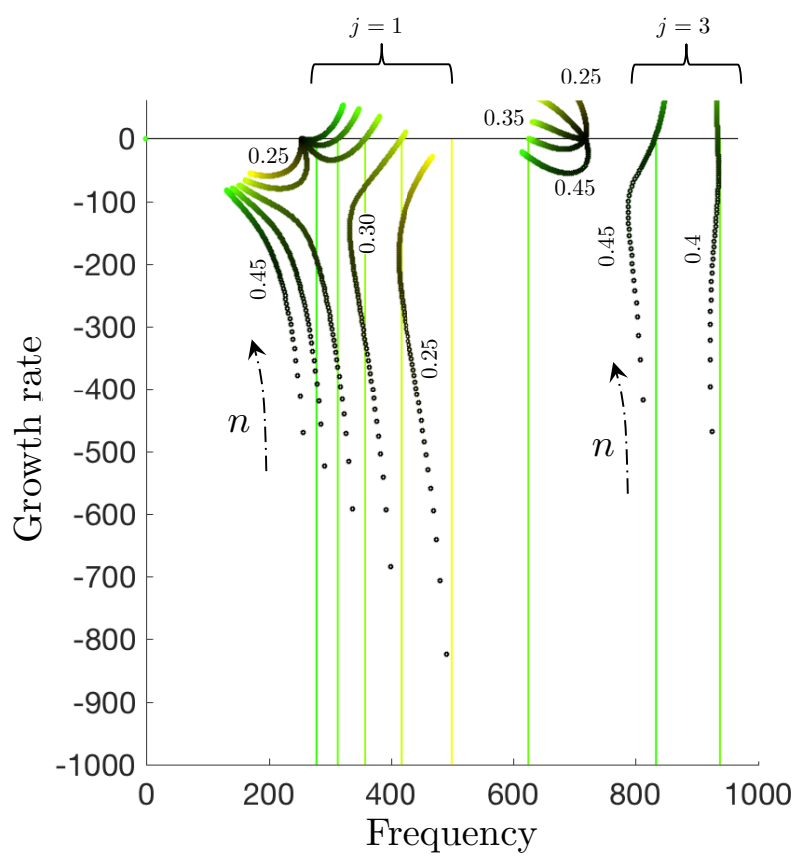

FIGURE 5. Locus of eigenfrequencies in the complex plane of the Duct configuration. $n=[0.005 \rightarrow 1]$ with $\Delta n=0.005$. Numbers in the plot are values of $m$ for the closest trajectory. Note that only the trajectories defined by $\tau=m \cdot 2 \pi / \omega_{1 p}$ with $m=0.25 \rightarrow 0.45(\Delta m=0.05)$ have been considered for readability. Vertical lines indicate frequencies equal to $j /(2 \tau)$.

Therefore, an explicit expression for the interaction index $n$ is found

$$
n=\frac{\left(\hat{p}_{k}^{\dagger}\right)^{H} \mathscr{A} \hat{p}_{k}+\omega_{k}^{2}}{\left(\hat{p}_{k}^{\dagger}\right)^{H} \mathscr{H} \hat{p}_{k} e^{-i \omega_{k} \tau}} .
$$

\section{The calculation of the critical flame gain}

In this study we calculate the value of $n$ such that the eigenfrequency is marginal, i.e. it is equal to $\omega_{g_{0}}=2 \pi \cdot j /(2 \tau)+i \cdot 0$. Therefore, in addition to $\mathscr{A}$ and $\mathscr{H}$, we know the quantities $\omega_{k}=\omega_{g_{0}}$ and $\tau$. In contrast, the eigenvectors $\hat{p}_{k}$ and $\hat{p}_{k}^{\dagger}$ at the neutral curve still have to be evaluated. In principle, we must know $n$ in order to solve the direct and adjoint eigenvalue problems given by Eqns. (8) and (9), respectively. We circumvent this difficulty as follows.

We re-write the active thermoacoustic system of Eqn. (8) as

$$
\mathscr{A} \hat{p}_{k}-\sigma_{k} \hat{p}_{k}=\underbrace{\mathscr{H} \mathscr{F}(\omega) \hat{p}_{k}}_{s_{a}}
$$

where $s_{a}$ is a vector acting as a forcing term at the region of the flame. Since we do not know the value of the vector $s_{a}$, we choose a vector $s$ with uniform forcing (and trivial value different from zero) at the region of the flame. We choose $s=I_{\mathrm{d}}$, i.e. a vector that is equal to one at the region of the flame and zero elsewhere. Consequently, the vector $s$ is proportional to $s_{a}$. The solution of Eqn. (12) with forcing term $s$ is denoted $\tilde{\hat{p}}_{k}$, and is considered proportional to $\hat{p}_{k}$ as explained in more detail in Appendix A. By carrying out a similar analysis we obtain $\tilde{\hat{p}}_{k}^{\dagger}$, which is also assumed proportional to $\hat{p}_{k}^{\dagger}$. Finally, the estimated direct $\hat{p}_{k}$ and adjoint eigenvectors $\hat{p}_{k}^{\dagger}$ are obtained by normalizing $\tilde{\hat{p}}_{k}$ and $\tilde{\hat{p}}_{k}^{\dagger}$, so that $\left(\hat{p}_{k}^{\dagger}\right)^{H} \hat{p}_{k}=1$ as explained in Appendix A.

\section{Sensitivity study}

In addition to estimating $n_{g_{0}}$ from Eqn. (11), we evaluate $\partial \omega /\left.\partial n\right|_{\omega_{g_{0}}}$, which is the sensitivity of $\omega$ with respect to small changes of $n$ around the critical flame gain, $n_{g_{0}}$. By applying the chain rule, we obtain

$$
\left.\frac{\partial \omega}{\partial n}\right|_{\omega_{g_{0}}}=\left(\frac{\partial \omega}{\partial \sigma} \frac{\partial \sigma}{\partial n}\right)_{\omega_{g_{0}}} \quad \text { where } \quad \frac{\partial \omega}{\partial \sigma}=\frac{-1}{2 \omega} .
$$

Following [22,23] and Silva et al. [25], we estimate $\partial \sigma / \partial n$ by means of a first-order adjoint formulation, which reads

$$
\frac{\partial \sigma}{\partial n}=\frac{\left(\hat{p}_{k}^{\dagger}\right)^{H} \frac{\partial(\mathscr{L}-I \sigma)}{\partial n} \hat{p}_{k}}{\left(\hat{p}_{k}^{\dagger}\right)^{H} \frac{\partial(\mathscr{L}-I \sigma)}{\partial \sigma} \hat{p}_{k}}=\frac{\left(\hat{p}_{k}^{\dagger}\right)^{H} \mathscr{H} \hat{p}_{k} e^{-i \omega \tau}}{\left(\hat{p}_{k}^{\dagger}\right)^{H} \mathscr{H} \hat{p}_{k} \frac{i n \tau}{2 \omega} e^{-i \omega \tau}+1}
$$

\section{Results}

We concentrate on two eigenfrequencies with zero growth rate defined as $\omega_{g_{0}}=2 \pi \cdot 1 /(2 \tau)+i \cdot 0$ where $\tau=m \cdot 2 \pi / \omega_{1 p}$. The corresponding cases are denoted D1 $(m=0.3)$ and D2 $(m=$ $0.4)$ for the Duct configuration and B1 $(m=0.3)$ and B2 $(m=$ 0.4 ) for the BRS combustor. These eigenfrequencies (crosses in Figs. 3 and 4) are arbitrarily chosen from the locus plot. It should be mentioned, however, that case B1 is related to the ITA instability observed in $[18,21]^{1}$. Following the procedure described in the previous section, we solve the linear systems

$$
\mathscr{A} \tilde{\hat{p}}_{k}-\sigma_{k} \tilde{\hat{p}}_{k}=I_{\mathrm{d}} \quad \text { and } \quad \mathscr{A}^{H} \tilde{\hat{p}}_{k}^{\dagger}-\sigma_{k}^{*} \tilde{\hat{p}}_{k}^{\dagger}=I_{\mathrm{d}}
$$

\footnotetext{
${ }^{1}$ The resonance frequency of case B1 in Fig. 4 is around $92 \mathrm{~Hz}$. In the studies of $[18,21]$ the ITA instability occurs at $98 \mathrm{~Hz}$. This difference in frequency is due to the slightly different outlet acoustic reflection and flame response used in this paper.
} 
where $\sigma_{k}=\sigma_{k}^{*}=-\omega_{g_{0}}^{2}$. Subsequently, the solutions $\tilde{\hat{p}}_{k}$ and $\tilde{\hat{p}}_{k}^{\dagger}$ are normalized by means of Eqn. (26) to obtain a first estimation of $\hat{p}$ and $\hat{p}^{\dagger}$. Results are shown in gray in Figs. (6) and (7) (although only distinguishable for $\hat{p}_{k}^{\dagger}$ in the region downstream of the flame). Next, we solve Eqn. (11) to obtain a first estimation of $n_{g_{0}}$. In order to obtain a better approximation of $\hat{p}$ and $\hat{p}^{\dagger}$, we solve

$$
\mathscr{L} \tilde{\hat{p}}_{k}-\sigma_{k} \tilde{\hat{p}}_{k}=I_{\mathrm{d}} \quad \text { and } \quad \mathscr{L}^{H} \tilde{\hat{p}}_{k}^{\dagger}-\sigma_{k}^{*} \tilde{\hat{p}}_{k}^{\dagger}=I_{\mathrm{d}}
$$

where $\sigma_{k}=\sigma_{k}^{*}=-\omega_{g_{0}}^{2}$ and the values of $n_{g_{0}}$ obtained previously are considered. Results are shown in Figs. (6) and (7) (in black although indistinguishable). These newly evaluated eigenvectors $\hat{p}_{k}$ and $\hat{p}_{k}^{\dagger}$ are used to solve again Eqn. (11) to revaluate $n_{g_{0}}$. Table 2 shows the values of $n_{g_{0}}$ for the first and second iterations. In order to validate the estimated interaction index $n_{g_{0}}$ and the corresponding eigenvectors $\hat{p}_{k}$ and $\hat{p}_{k}^{\dagger}$, we solve the nonlinear eigenvalue problem given by Eqn. (9) accounting for the estimated value $n_{g_{0}}$ in the second iteration. Results are displayed in Figs. (6) and (7) (dashed blue and red lines). It is interesting to observe that the acoustic field related to $\hat{p}_{k}$ is well estimated from the first iteration, and therefore gray, black and blue lines are exactly superposed. In addition, we see that the value of the eigenfrequency $\omega_{k}$ obtained by solving Eqn. (8) with the evaluated $n_{g_{0}}$ is also close to the one given by $\omega_{g_{0}}$, as shown in Tab. 3 . This verifies the proposed algorithm.

TABLE 2. Values of $n_{g_{0}}$ obtained in first and second iterations for the four cases under investigation.

\begin{tabular}{|l|l|c|c|c|c|}
\hline Parameter & Iteration & D1 & D2 & B1 & B2 \\
\hline$n_{g_{0}}$ & 1 & 0.718 & 0.346 & 3.147 & 1.703 \\
\hline$n_{g_{0}}$ & 2 & 0.723 & 0.353 & 3.156 & 1.718 \\
\hline
\end{tabular}

TABLE 3. Eigenfrequencies under consideration

\begin{tabular}{|l|l|l|}
\hline Case & $\omega_{g_{0}}=2 \pi \cdot 1 /(2 \tau)+i \cdot 0$ & $\omega_{k}$ (from Eqn. (9) ) \\
\hline D1 & $2 \pi \cdot 416.667+0 \mathrm{i}$ & $2 \pi \cdot 416.667-0.00089 \mathrm{i}$ \\
\hline $\mathrm{D} 2$ & $2 \pi \cdot 312.5+0 \mathrm{i}$ & $2 \pi \cdot 312.485-0.004 \mathrm{i}$ \\
\hline $\mathrm{B} 1$ & $2 \pi \cdot 91.667+0 \mathrm{i}$ & $2 \pi \cdot 91.667+0 \mathrm{i}$ \\
\hline B2 & $2 \pi \cdot 68.75+0 \mathrm{i}$ & $2 \pi \cdot 68.75+0 \mathrm{i}$ \\
\hline
\end{tabular}
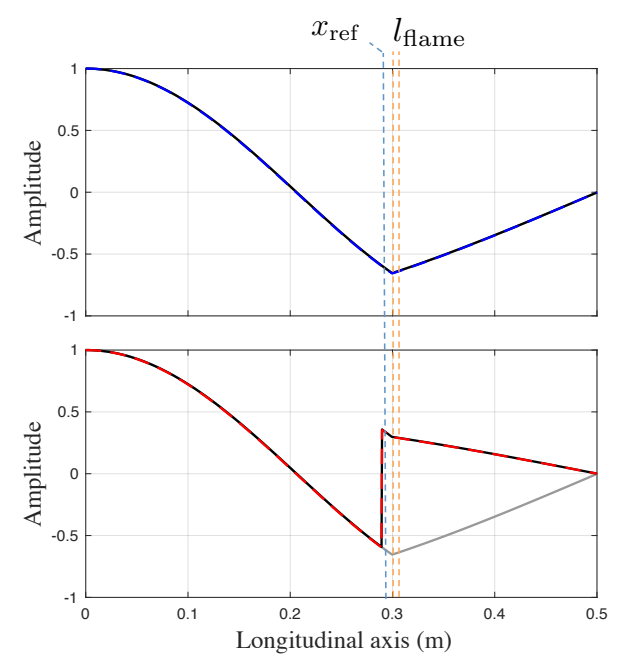

FIGURE 6. Thermoacoustic modes $\hat{p} / \max (\hat{p})$ (top) and $\hat{p}^{\dagger} / \max \left(\hat{p}^{\dagger}\right)$ (bottom) of the Duct configuration (D1). (Gray) first iteration, (Black) second iteration, (Dashed blue) $\hat{p}_{k}$ from direct eigenvalue problem of Eqn. (8). (Dashed red) $\hat{p}_{k}^{\dagger}$ from adjoint eigenvalue problem of Eqn. (9). Note that gray and black curves overlap the blue and red curves, which verifies the proposed algorithm.

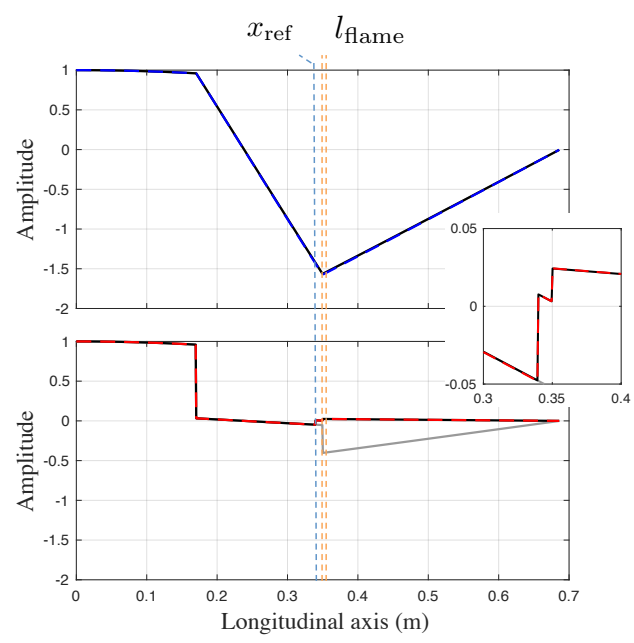

FIGURE 7. Thermoacoustic modes $\hat{p} / \max (\hat{p})$ (top) and $\hat{p}^{\dagger} / \max \left(\hat{p}^{\dagger}\right)$ (bottom) of the BRS configuration (B1). (Gray) first iteration, (Black) second iteration, (Dashed blue) $\hat{p}_{k}$ from direct eigenvalue problem of Eqn. (8). (Dashed red) $\hat{p}_{k}^{\dagger}$ from adjoint eigenvalue problem of Eqn. (9). Note that gray and black curves overlap the blue and red curves, which verifies the proposed algorithm.

At the region of marginal stability, it is important not only to know the critical values for the interaction index $n_{g_{0}}$, but also 
to calculate the sensitivity of the eigenfrequency for a small change in $n$. Therefore, we apply Eqns. (13) and (14) to calculate $\partial \omega /\left.\partial n\right|_{\omega_{g_{0}}}$ using the eigenvectors $\hat{p}_{k}$ and $\hat{p}_{k}^{\dagger}$ obtained in the second iteration of the method proposed. The sensitivity calculated with the adjoint method exactly matches the slope of the curve (Figs. 8 and 9). In other words, it is first-order accurate. Such an adjoint sensitivity study can be carried out for any other system's parameter, such as time delay $\tau$ or acoustic reflection at the boundaries, at zero extra cost.

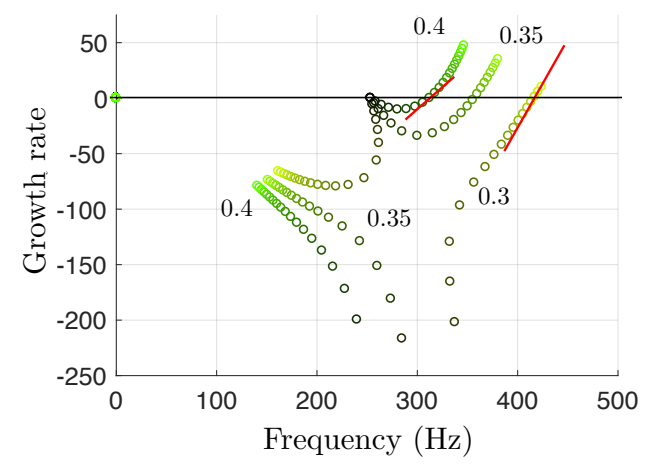

FIGURE 8. Locus of eigenfrequencies in the complex plane of the Duct configuration. Note that this figure is an extract of Fig. 3. The red lines indicate the slope (sensitivity $\partial \omega /\left.\partial n\right|_{\omega_{g_{0}}}$ ) computed with the adjoint method. The growth rate is defined as $-\operatorname{Im}(\omega) / 2 \pi$.

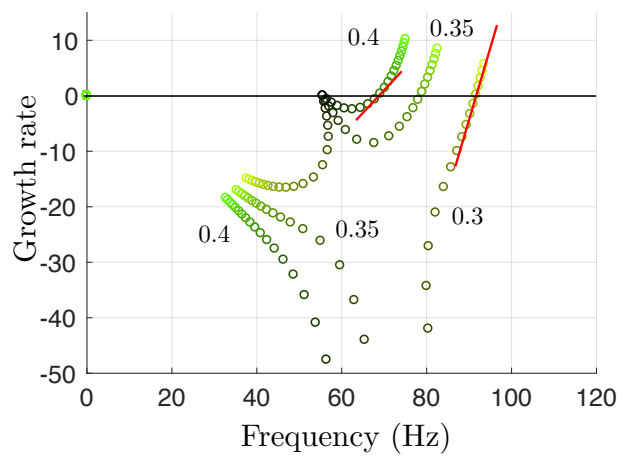

FIGURE 9. Locus of eigenfrequencies in the complex plane of the BRS configuration. Note that this figure is an extract of Fig. 4. The red lines indicate the slope (sensitivity $\partial \omega /\left.\partial n\right|_{\omega_{g_{0}}}$ ) computed with the adjoint method. The growth rate is defined as $-\operatorname{Im}(\omega) / 2 \pi$.

\section{Main parameters affecting the critical gain}

By studying Eqn. (11), it is possible to identify which thermodynamic and geometric parameters can make a combustor prone to thermoacoustic instabilities, i.e. under which conditions Eqn. (11) gives low values of $n_{g_{0}}$. Letting aside the influence of $\omega$ and $\tau$, we realize that a combustor described by the terms $\left(\hat{p}_{k}^{\dagger}\right)^{H} \mathscr{A} \hat{p}_{k}$ and $\left(\hat{p}_{k}^{\dagger}\right)^{H} \mathscr{H} \hat{p}_{k}$ with low and large values, respectively, is prone to exhibit thermoacoustic instability for low values of the interaction index $n$.

We first analyze the term $\left(\hat{p}_{k}^{\dagger}\right)^{H} \mathscr{A} \hat{p}_{k}$ for the four cases investigated, whose values are shown in Tab. 4 . When comparing D1 against B1 and D2 against B2, it is observed that low values of this term do not imply necessarily low values of $n_{g_{0}}$ (last column of Tab. 4). Therefore, analyzing $\left(\hat{p}_{k}^{\dagger}\right)^{H} \mathscr{A} \hat{p}_{k}$ does not give clues about which parameters control the stability of the combustors investigated. Therefore, we analyze the term

$$
\mathscr{T}=\left(\hat{p}_{k}^{\dagger}\right)^{H} \mathscr{H} \hat{p}_{k}=\left(\hat{p}_{k}^{\dagger}\right)^{H} I_{\mathrm{d}} \theta \bar{c}_{\mathrm{ref}}^{2} S_{\mathrm{ref}} /\left.\left(S_{\mathrm{f}} l_{\mathrm{f}}\right) \frac{\partial \hat{p}_{k}}{\partial x}\right|_{\text {ref }}
$$

to evaluate under which conditions it has large values. By looking at Eqn. (17) we realize that $\mathscr{T}$ is proportional to $\theta, \bar{c}_{\text {ref }}^{2}$, $S_{\text {ref }} / S_{\mathrm{f}}$ and $1 / l_{\mathrm{f}}$. The corresponding values for the Duct and BRS configurations are displayed in Tab. 5. The parameters $\theta, \bar{c}_{\text {ref }}$ and $l_{\mathrm{f}}$ have similar values for both BRS and duct systems. In contrast, we observe that the area ratio $S_{\text {ref }} / S_{\mathrm{f}}$ associated with the Duct configuration is approximately one order of magnitude larger than the one corresponding to the BRS combustor. This result implies that low values of $S_{\text {ref }} / S_{\mathrm{f}}$ contribute to the stability conditioning of a combustor. Another factor of high impact on the value of $\mathscr{T}$ is the product $\left(\hat{p}_{k}^{\dagger}\right)^{H} I_{\mathrm{d}} \partial \hat{p}_{k} /\left.\partial x\right|_{\text {ref }}$. This parameter multiplies the value of $\hat{p}_{k}^{\dagger}$ at the flame region by the value of $\partial \hat{p}_{k} / \partial x$ at the reference position. Its value is given in Tab. 4 for the four cases under study. The Duct configuration through cases $\mathrm{D} 1$ and D2 exhibits values of $\left(\hat{p}_{k}^{\dagger}\right)^{H} I_{\mathrm{d}} \partial \hat{p}_{k} /\left.\partial x\right|_{\text {ref }}$ which are almost two order of magnitudes larger than the counterparts B1 and B2 of the BRS combustor. The fact that the Duct combustor has smaller critical gains than the BRS combustor explains why the former is more prone to be thermoacoustically unstable than the latter.

We conclude the study by highlighting the importance of the parameter $\mathscr{T}$, which in one term summarizes the contributions of all thermodynamic and geometric parameters on the stability of a quasi-1D thermoacoustic system. We realize also that this parameter plays a crucial role in the description of $\partial \omega /\left.\partial n\right|_{\omega_{g_{0}}}$ as evidenced in Eqn. (14). The present study can be extended to three-dimensional combustors, where, as performed in the present work, the influence of flame location-distribution and combustor geometry on the stability of the system are encapsulated in the operator $\mathscr{H}$ and both direct and adjoint eigenvectors. 


\begin{tabular}{|l|c|c|c|} 
TABLE 4. Parameters of interest \\
\begin{tabular}{|l|c|c|c|}
\hline Parameter & $\left(\hat{p}_{k}^{\dagger}\right)^{H} \mathscr{A} \hat{p}_{k}$ & $\left.\left(\hat{p}_{k}^{\dagger}\right)^{H} I_{\mathrm{d}} \frac{\partial \hat{p}_{k}}{\partial x}\right|_{\mathrm{ref}}$ & $n_{g_{0}}$ \\
\hline D1 & $2.4119 \mathrm{e}+05$ & -0.0187 & 0.723 \\
\hline B1 & $2.2442 \mathrm{e}+05$ & -0.0028 & 3.156 \\
\hline \hline D2 & $-1.8196 \mathrm{e}+06$ & -0.0110 & 0.353 \\
\hline B2 & $-8.9071 \mathrm{e}+04$ & $-9.09 \cdot 10^{-4}$ & 1.718 \\
\hline
\end{tabular}
\end{tabular}

TABLE 5. Parameters influencing the term $\mathscr{T}$

\begin{tabular}{|l|c|c|}
\hline Parameter & BRS & Duct \\
\hline$\theta$ & 5.5870 & 4.4608 \\
\hline$l_{\mathrm{f}}$ & 0.0014 & 0.001 \\
\hline $\bar{c}_{\text {ref }}^{2}$ & 117730 & 117810 \\
\hline$S_{\text {ref }} / S_{\mathrm{f}}$ & 0.1303 & 1 \\
\hline
\end{tabular}

\section{CONCLUSIONS}

The thermoacoustic modes at the region of marginal stability of two different quasi-1D combustors with fully acoustically reflecting boundary conditions were investigated. The trajectories of the eigenfrequencies in the complex plane were calculated as a function of the flame gain, $n$, and time delay, $\tau$. It was found that the eigenfrequency associated with the active thermoacoustic modes at the neutral curve depends only on the flame dynamics, i.e. it is independent of the geometry of the combustor. Furthermore, it was noted that the loci of eigenfrequencies of two different combustors are qualitatively similar. This finding is of relevance when characterizing the acoustic activity of an unstable combustor, where resonance frequencies may be related not only to (perturbed) passive thermoacoustic modes but also to ITA modes.

In the second part of the article, an adjoint-based method is proposed to accurately evaluate at a low computational cost the critical gain and corresponding sensitivity at the neutral curve. The methodology may be used to quantify how prone a given combustor is to exhibit combustion instabilities. For example, large values of the critical gain (for a given value of $\tau$ ) would imply a robustly stable combustor, because a strong flame (high gain) would be necessary for a instability to be triggered.

It was found that the calculation of the eigenfrequencies of the quasi-vertical (ITA) trajectories, whose decay rates tend to infinity as $n$ decreases, is a computationally challenging task if a fixed-point iteration is deployed. Indeed, it was necessary to significantly lower the value of the relaxation coefficient to make the iterations converge. This may be one of the reasons why Helmholtz solvers have not been yet widely used for the study of
ITA modes. With the adjoint-based method proposed, it is possible to accurately calculate the marginally stable eigenfrequencies and the corresponding thermoacoustic modes with cheap calculations.

\section{ACKNOWLEDGMENT}

Luca Magri gratefully acknowledges the support from the Royal Academy of Engineering Research Fellowships Scheme. The authors acknowledge fruitful discussions with Prof. Jonas Moeck and Georg Mensah on the defective eigenvalue. Camilo Silva acknowledges the work of Robin Caloudis in the code development that enormously facilitated the execution of this study.

\section{REFERENCES}

[1] Lieuwen, T., and Yang V., (Eds.), 2005. "Combustion instabilities in gas turbine engines: operational experience, fundamental mechanisms and modeling". Prog. in Astronautics and Aeronautics AIAA, 210.

[2] Candel, S., 2002. "Combustion dynamics and control: Progress and challenges". Proc. Combust. Inst., 29, pp. 128.

[3] Durox, D., Schuller, T., Noiray, N., and Candel, S., 2009. "Experimental analysis of nonlinear flame transfer functions for different flame geometries". Proc. Combust. Inst., 32, pp. 1391-1398.

[4] Kim, K., Lee, J., Quay, B., and Santavicca, D., 2010. "Spatially distributed flame transfer functions for predicting combustion dynamics in lean premixed gas turbine combustors". Combust. Flame, 157, pp. 1718-1730.

[5] Tay-Wo-Chong, L., Bomberg, S., Ulhaq, A., Komarek, T., and Polifke, W., 2012. "Comparative validation study on identification of premixed flame transfer function". J. Eng. Gas Turb. and Power, 134(2), pp. 021502-1-8.

[6] Silva, C. F., Emmert, T., Jaensch, S., and Polifke, W., 2015. "Numerical study on intrinsic thermoacoustic instability of a laminar premixed flame". Combust. Flame , 162, pp. 3370-3378.

[7] Keller, J. J., 1995. "Thermoacoustic Oscillations in Combustion Chambers of Gas Turbines". AIAA Journal, 33(12), pp. 2280-2287.

[8] Nicoud, F., Benoit, L., and Sensiau, C., 2007. "Acoustic modes in combustors with complex impedances and multidimensional active flames". AIAA Journal, 45, pp. 426441.

[9] Silva, C. F., Nicoud, F., Schuller, T., Durox, D., and Candel, S., 2013. "Combining a Helmholtz solver with the flame describing function to assess combustion instability in a premixed swirled combustor". Combust. Flame, 160(9), pp. 1743-1754.

[10] Emmert, T., Meindl, M., Jaensch, S., and Polifke, W., 2016. 
"Linear State Space Interconnect Modeling of Acoustic Systems". Acta Acustica united with Acustica, 102(5), pp. 824-833.

[11] Yi, T., and Gutmark, E. J., 2008. "Online prediction of the onset of combustion instability based on the computation of damping ratios". Journal of Sound and Vibration, 310(1), pp. 442-447.

[12] Crocco, L., 1951. "Aspects of combustion instability in liquid propellant rocket motors. Part I". J. American Rocket Society, 21, pp. 163-178.

[13] Crocco, L., 1952. "Aspects of combustion instability in liquid propellant rocket motors. Part II". J. American Rocket Society, 22, pp. 77-16.

[14] Poinsot, T., and Veynante, D., 2012. Theoretical and numerical combustion. R. T. Edwards.

[15] Hoeijmakers, M., Kornilov, V., I. Lopez Arteaga a, d. P. d. G., and Nijmeijer, H., 2014. "Intrinsic instability of flame-acoustic coupling". Combust. Flame, 161(11), pp. 2860-2867.

[16] Emmert, T., Bomberg, S., and Polifke, W., 2015. "Intrinsic thermoacoustic instability of premixed flames". Combust. Flame, 162(1), pp. 75-85.

[17] Courtine, E., Selle, L., and Poinsot, T., 2015. "DNS of Intrinsic Thermoacoustic Modes in Laminar Premixed Flames". Combustion and Flame, 162(11), pp. 4331-4341.

[18] Silva, C. F., Merk, M., Komarek, T., and Polifke, W., 2017. "The Contribution of Intrinsic Thermoacoustic Feedback to Combustion Noise and Resonances of a Confined Turbulent Premixed Flame". Combustion and Flame, 182, pp. 269278. bibtex: SilvaMerk17.

[19] Mukherjee, N., and Shrira, V., 2017. "Intrinsic Flame Instabilities in Combustors: Analytic Description of a 1-D Resonator Model". Combustion and Flame, 185, pp. 188-209. bibtex: MukheShrir17.

[20] Komarek, T., and Polifke, W., 2010. "Impact of swirl fluctuations on the flame response of a perfectly premixed swirl burner". J. Eng. Gas Turbines Power, 132(6), June, pp. 061503-1,7.

[21] Albayrak, A., Steinbacher, T., Komarek, T., and Polifke, W., 2017. "Convective Scaling of Intrinsic ThermoAcoustic Eigenfrequencies of a Premixed Swirl Combustor". J. Eng. Gas Turbines and Power.

[22] Magri, L., and Juniper, M. P., 2013. "Sensitivity analysis of a time-delayed thermo-acoustic system via an adjoint-based approach”. Journal of Fluid Mechanics, 719, pp. 183-202.

[23] Magri, L., Bauerheim, M., and Juniper, M. P., 2016. "Stability analysis of thermo-acoustic nonlinear eigenproblems in annular combustors. Part I. Sensitivity". Journal of Computational Physics, 325, pp. $395-410$.

[24] Magri, L., and Juniper, M. P., 2014. "Adjoint-based linear analysis in reduced-order thermo-acoustic models". Int. J. Spray Combust. Dyn., 6(3), pp. 225-246.
[25] Silva, C., Magri, L., Runte, T., and Polifke, W., 2017. "Uncertainty quantification of growth rates of thermoacoustic instability by an adjoint Helmholtz solver". J. Eng. Gas Turbines and Power, 139(1).

\section{A Relation between $\hat{p}_{k}$, $\hat{p}_{k}^{\dagger}$ and $\tilde{\hat{p}}_{k}$, $\tilde{\hat{p}}_{k}^{\dagger}$}

The Helmholtz Equation reads

$$
\mathscr{A} \hat{p}_{k}+\omega_{k}^{2} \hat{p}_{k}-\mathscr{H} \mathscr{F}(\omega) \hat{p}_{k}=0
$$

where $\hat{p}_{k}$ and $\omega_{k}^{2}$ are the $k_{\mathrm{th}}$ eigenvector and eigenfrequency of the active thermoacoustic system. We now assume that the active acoustic eigenvectors can be well represented by a linear combination of the passive acoustic eigenvectors as $\hat{p}_{k}=\sum_{k=1}^{N} \hat{p}_{k p} \eta_{k_{p}}$, where $N$ is the number of modes considered and $\eta_{k_{p}}$ are weighting coefficients. Equation (18) reads now

$$
\mathscr{A} \sum_{k=1}^{N} \hat{p}_{k_{p}} \eta_{k_{p}}+\omega_{k}^{2} \sum_{k=1}^{N} \hat{p}_{k p} \eta_{k_{p}}-\mathscr{H} \mathscr{F}(\omega) \sum_{k=1}^{N} \hat{p}_{k p} \eta_{k_{p}}=0
$$

By recalling that the passive eigenvalue problem is defined by $\mathscr{A} \hat{p}_{k_{p}}=-\omega_{k_{p}}^{2} \hat{p}_{k_{p}}$, we rewrite Eqn. (19) as

$$
-\sum_{k=1}^{N} \hat{p}_{k_{p}} \omega_{k_{p}}^{2} \eta_{k_{p}}+\omega_{k}^{2} \sum_{k=1}^{N} \hat{p}_{k p} \eta_{k_{p}}-\mathscr{H} \mathscr{F}(\omega) \sum_{k=1}^{N} \hat{p}_{k p} \eta_{k_{p}}=0
$$

By exploiting the biorthogonality between $\hat{p}_{k_{p}}$ and $\hat{p}_{k_{p}}^{\dagger}$, which means $\left(\hat{p}_{k_{p}}^{\dagger}\right)^{H} \hat{p}_{l_{p}}=\delta_{k l}$, we project Eqn. (20) on to the basis of the adjoint eigenvectors. Equation (20) becomes

$$
\omega_{k}^{2} \eta_{k_{p}}-\omega_{k_{p}}^{2} \eta_{k_{p}}-\left(\hat{p}_{k_{p}}^{\dagger}\right)^{H} \underbrace{\mathscr{H} \mathscr{F}(\omega) \sum_{k=1}^{N} \hat{p}_{k p} \eta_{k_{p}}}_{s_{a}}=0
$$

We consider now the last term $s_{a}$ of Eqn. (21) to be a forcing term that acts exclusively in the region of the flame. Rearranging Eqn. (21), we obtain

$$
\eta_{k_{p}}=\frac{\left(\hat{p}_{k_{p}}^{\dagger}\right)^{H} s_{a}}{\omega_{k}^{2}-\omega_{k_{p}}^{2}} \quad \text { and subsequently } \quad \hat{p}_{k}=\sum_{k=1}^{N} \hat{p}_{k p} \eta_{k_{p}}
$$

The vector $s_{a}$ is unknown. Therefore, we consider a vector $s$ that acts as a uniform (with trivial value different from zero) forcing term in the region of the flame. In this study we set $s=I_{\mathrm{d}}$. The 
solution of this problem is given by

$$
\tilde{\eta}_{k_{p}}=\frac{\left(\hat{p}_{k_{p}}^{\dagger}\right)^{H} s}{\omega_{k}^{2}-\omega_{k_{p}}^{2}} \quad \text { and subsequently } \quad \tilde{\hat{p}}_{k}=\sum_{k=1}^{N} \hat{p}_{k p} \tilde{\eta}_{k_{p}}
$$

or directly by solving the linear system

$$
\mathscr{A} \tilde{\hat{p}}_{k}+\omega_{k}^{2} \tilde{\hat{p}}_{k}=s
$$

Note that the vectors $s_{a}$ and $s$ are proportional. As a result, the active acoustic eigenvector $\hat{p}_{k}$ can be considered proportional to $\tilde{\hat{p}}_{k}$. This can be understood if comparing Eqn. (22) to Eqn. (23). By carrying out a similar analysis, we can find $\tilde{\hat{p}}^{\dagger}$ by solving

$$
\mathscr{A}^{H} \tilde{\tilde{p}}^{\dagger}+\omega_{k}^{* 2} \tilde{\hat{p}}^{\dagger}=s
$$

where $\tilde{\hat{p}}^{\dagger}$ is considered proportional to the adjoint eigenvector $\hat{p}^{\dagger}$. We need to find now a correct scaling. This is done by normalizing $\tilde{\hat{p}}$ and $\tilde{\hat{p}}^{\dagger}$ as

$$
\hat{p}_{k}=\frac{\tilde{\hat{p}}_{k}}{\sqrt{\left(\tilde{\hat{p}}_{k}^{\dagger}\right)^{H} \tilde{\hat{p}}_{l}}} \quad \text { and } \quad \hat{p}_{k}^{\dagger}=\frac{\tilde{\hat{p}}_{k}^{\dagger}}{\sqrt{\left(\tilde{\hat{p}}_{k}^{\dagger}\right)^{H} \tilde{\hat{p}}_{l}}}
$$

so that $\left(\hat{p}_{k}^{\dagger}\right)^{H} \hat{p}_{k}=1$ is satisfied. 